www.conferenceie.ase.ro

\title{
A.I. NEURAL NETWORKS INFERENCE INTO THE IOT EMBEDDED DEVICES USING TINYML FOR PATTERN DETECTION WITHIN A SECURITY SYSTEM
}

\author{
Cristian TOMA \\ Department of Economic Informatics \& Cybernetics \\ Bucharest University of Economic Studies, Romania \\ cristian.toma@ie.ase.ro \\ Marius POPA \\ Department of Economic Informatics \& Cybernetics \\ Bucharest University of Economic Studies, Romania \\ marius.popa@ie.ase.ro \\ Mihai DOINEA \\ Department of Economic Informatics \& Cybernetics \\ Bucharest University of Economic Studies, Romania \\ mihai.doinea@ie.ase.ro
}

\begin{abstract}
The paper presents the implementation challenges of a proof of concept development for image processing with artificial intelligence neural network into an embedded device. Because of the hardware constraints of the embedded device - e.g. $16 \mathrm{~KB} R A M$ and $128 \mathrm{~KB}$ of EEPROM, the deep learning/the training and the model of the neural network is processed in cloud-e.g. Google Cloud Platform-AI-Artificial intelligence, as shown in second section. After this step, the trained neural networks model, values and tensors are translated with TinyML into native code for the embedded devices and deployed on a specific hardware platform - e.g. Arduino Nano 33 BLE Sense or SparkFun Edge Development Board Apollo3 Blue - for the neural network inferences - e.g. person detection or NLP into specific area, as described in third section. The last section shows the conclusions and the security challenges for deploying neural networks into embedded systems which are used for security systems such as: monitoring and surveillance cameras, drones visual computing for securing field areas, IoT systems, etc.
\end{abstract}

Keywords: Artificial Intelligence, Neural Networks, Deep Learning, embedded devices, TinyML, cybersecurity, IoT.

JEL classification: C88, L86, Y80

DOI: $10.24818 / \mathrm{ie} 2020.01 .03$

\section{Introduction into Neural Networks Inferences Process}

There are multiple software development kits, libraries and frameworks for developing Neural Networks and Deep Learning applications. Most of them are pushing Python based code, but Python has poor results for the performance benchmarking on real CPU and GPU cores. This maybe an advantage for the cloud providers but not necessary an advantage for the companies or software integrators who are renting processing power into the Cloud. Most used framework/API is Keras. are which can be accessible from the Keras API. Keras API may address via API multi-back-end Artificial Intelligence Cloud solutions, such as: Tensorflow, Theano, MxNet. Tensorflow is polyglot and is supporting Java and JVM based languages as well, but for the moment, Python is pushed into a lot of books and tutorials. Additionally, in 
the A.I field are various libraries/frameworks/even OS-es for dedicated processing from NLP - Natural Language Processing - e.g. Apache NLP to Visual Computing and generic deep learning - e.g. Eclipse deeplearning4j, Apache Spark or ROS - Robot Operating System.

Figure 1 presents a Java program which is implementing an Artificial Intelligence Feed Forward Neuronal Network with back-propagation for hand-writing recognition.

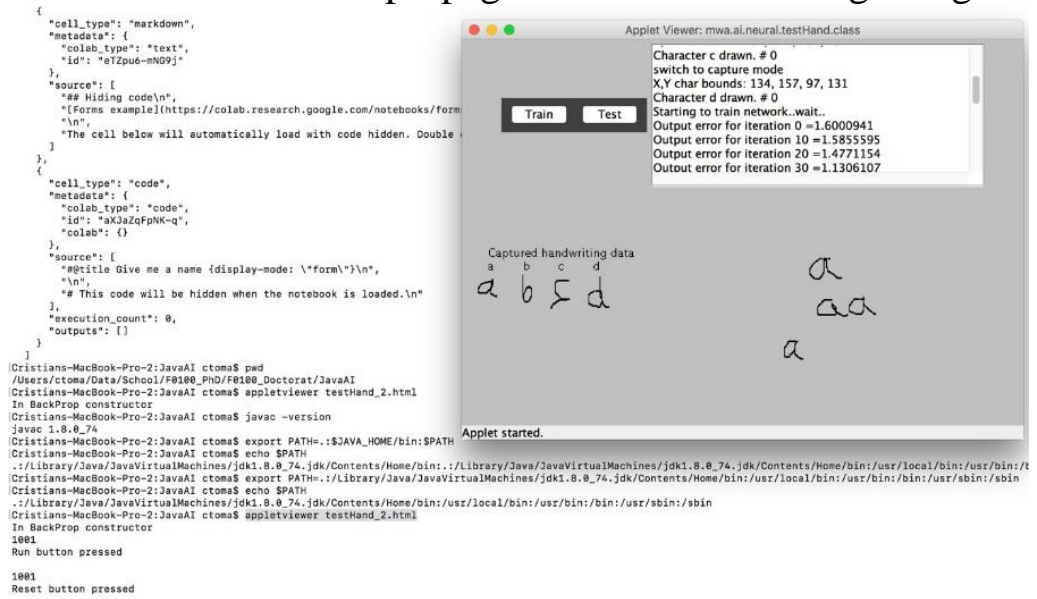

Figure 1. Java implementation using feed forward multi-layered ANN-Artificial Neuronal Network with back-

Input Canvas: propagation for hand-writing recognition

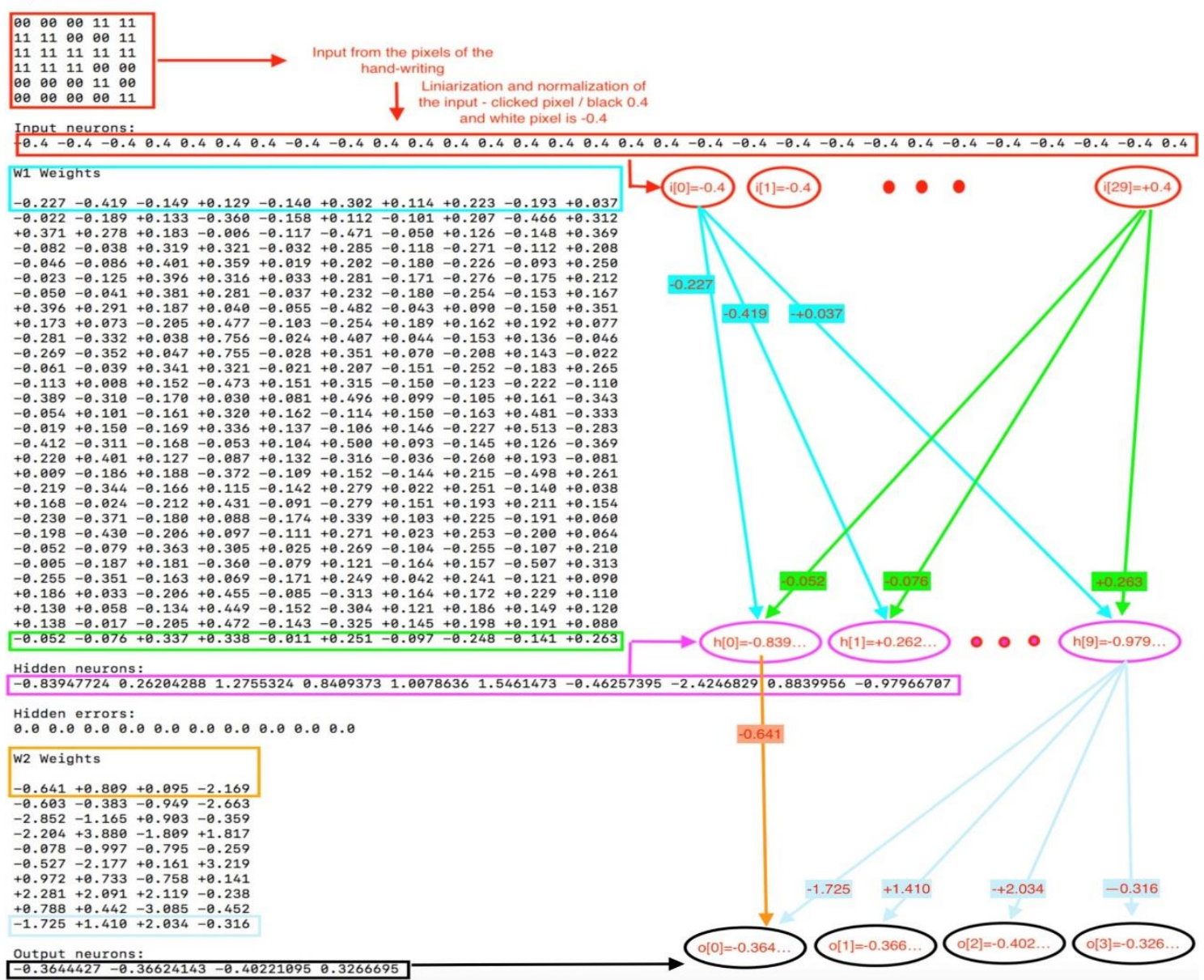

Figure 2. Neuronal network values for hidden layer and for weights matrixes

In figure 2 are shown for the program from Fig. 1 the neuronal network architecture/model, but with non-deterministic values, because of different data set used for the training process. 
The learning process is supervised in this use case and it is a non-deterministic process. After each set of iterations/epochs of training of the neuronal network, the model has different values for the hidden layers and weights. Once this values are set, the model/the neuronal network is ready to do inferences in a deterministic manner. The inference is the process for running new data sets within a trained neuronal network in order to obtain classifications based on the training process.

Because of the hardware constrains of the IoT Embedded devices, it impossible to do the training process within these devices, but the inference is possible:

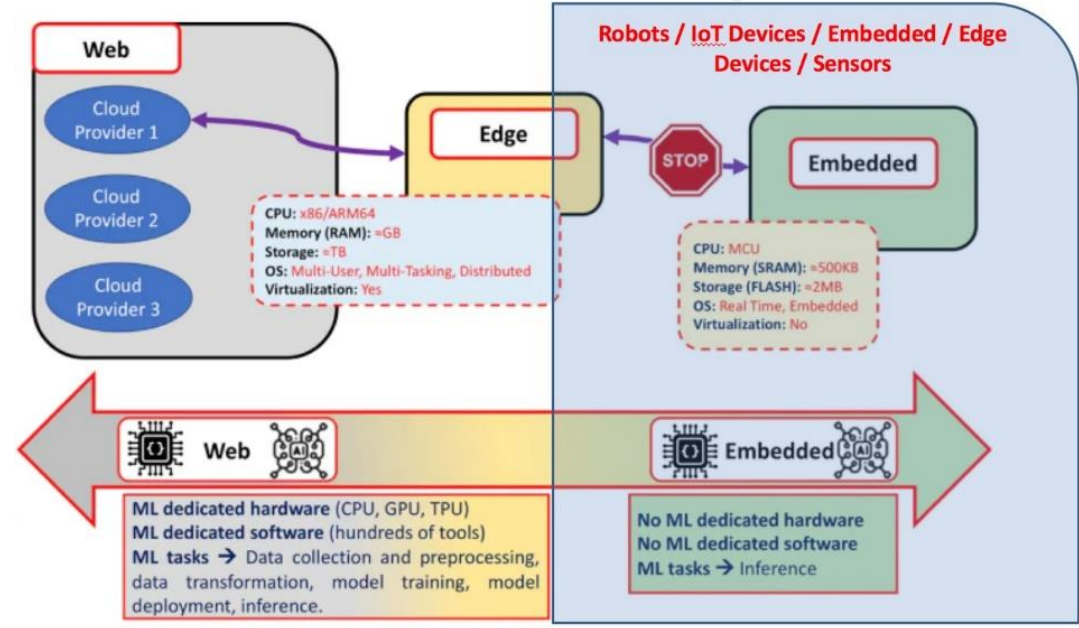

Figure 3. Only the Neuronal Network Inference process is run into embedded device - copyright [6]

Therefore, for the final inference process, because the IoT Nodes such as Arduino Nano 33 BLE Sense (Arduino platform), SparkFun Edge Development Board Apollo3 Blue, mbed LPC1768 (mbed OS), ST Microelectronics STM32F746G or ESP8266 embedded devices have $16 / 32 \mathrm{~KB}$ RAM and 128/512 KB of EEPROM, it is enough to store the values for the trained model - e.g. for feed-forward neural network with back propagation. The values from Fig. 2 can be stored in buffers inside NVM - Non Volatile Memory and they will be used for the inferences. In the next section there are details about the eco-system available in the cloud for training deep learning models and artificial intelligence neural networks by using CPUs, GPUs, TPUs and hardware dedicated accelerators for the machine learning algorithms.

\section{Development of Training Models in AI Clouds}

Briefly, an AI Cloud is a cloud infrastructure that provides AI services. The main reason to get cloud-based AI services is the cost to develop such products in-house. Hence, the demand has increased for outsourced AI services.

The main providers of cloud computing services includes AI services within their offers. The big players in the IT\&C industry have the knowledge and financial power to support development and deployment of such services for AI. The major cloud-based AI services providers and the main characteristics of AI packages are presented below:

- Amazon Web Services - provides services and tools both to develop, train and deploy custom machine learning models and use of some pre-trained AI services for customers in different areas like computer vision, language, recommendations and forecasting.

- Microsoft Azure - aims to achieve human capabilities in object and speech recognition and machine translation. Those goals are intended to be reached through machine learning, knowledge mining and AI applications and agents used to customize models with specific data and model deployment anywhere. 
- Google Cloud - facilitates custom AI application development by using machine learning tools and services to prepare data, build and run applications, manage the models and workflows, and share the machine learning pipelines with other users and to deploy them anywhere.

- IBM Cloud - provides a suite of services for AI, starting with Watson Studio for machine learning and deep learning, or by using specialized services and tools for visual recognition, speech to text, text to speech, language translator and so forth.

- Salesforce - is based on Einstein platform to create custom AI models for predictions, CRM-based bots, deep learning models for image recognition, language-based classifiers and data pattern models.

- Oracle AI - leverages AI in the cloud services to get businesses smarter by using platforms like Data Science, Embedded ML, Digital Assistant and DataFox.

- Alibaba Cloud - provides end-to-end machine learning services for data processing, data mining and analysis, and natural language processing.

- Baidu - performs investments and research efforts in different areas of AI like machine learning base products, natural language processing, smart homes, data science and automation tools.

However, the AI field is not accessible by any actor in ICT industry because of huge costs, especially the ones determined by the hardware infrastructure needed to support complex models and big data. The current challenge is to improve those AI cloud infrastructures to support new features determined by the demands of a new smarter world. Hence, fields like Internet of Things where tiny computers and devices are connected and used over internet together with collected and generated data has leaded to the need to apply AI features on such kind of tiny systems. The title [1] passes through AI processed developed for such tiny systems being wide-spread increasingly. Existing AI cloud infrastructures are used to achieve deployments on such tiny systems by using specific cloud services that allow AI deployments on embedded systems. An example of such cloud infrastructure is Google Cloud used in [1] also. Machine learning is a subfield of AI. It supposes passing a model through some steps according figure 4 presented at [8]. The components of AI platform are [7]:

- Training service - trains models with customizable options like distributed training, hyperparameter settings and usage of GPU (Graphics Processing Unit) or TPU (Tensor Processing Unit).

- Prediction service - make predictions on a trained model no mattering where the model was trained before.

- Notebooks - allow use of virtual machines prepackaged with JupyterLab. JupyterLab is a web-based interface facilitating work with documents and activities as Jupyter notebooks. Also, it provides a unified view of different file formats.

- Data labeling service - labels a dataset used for custom ML model.

- Deep learning VM image - provides the option to choose a virtual machine image customized with ML frameworks and tools. 
www.conferenceie.ase.ro

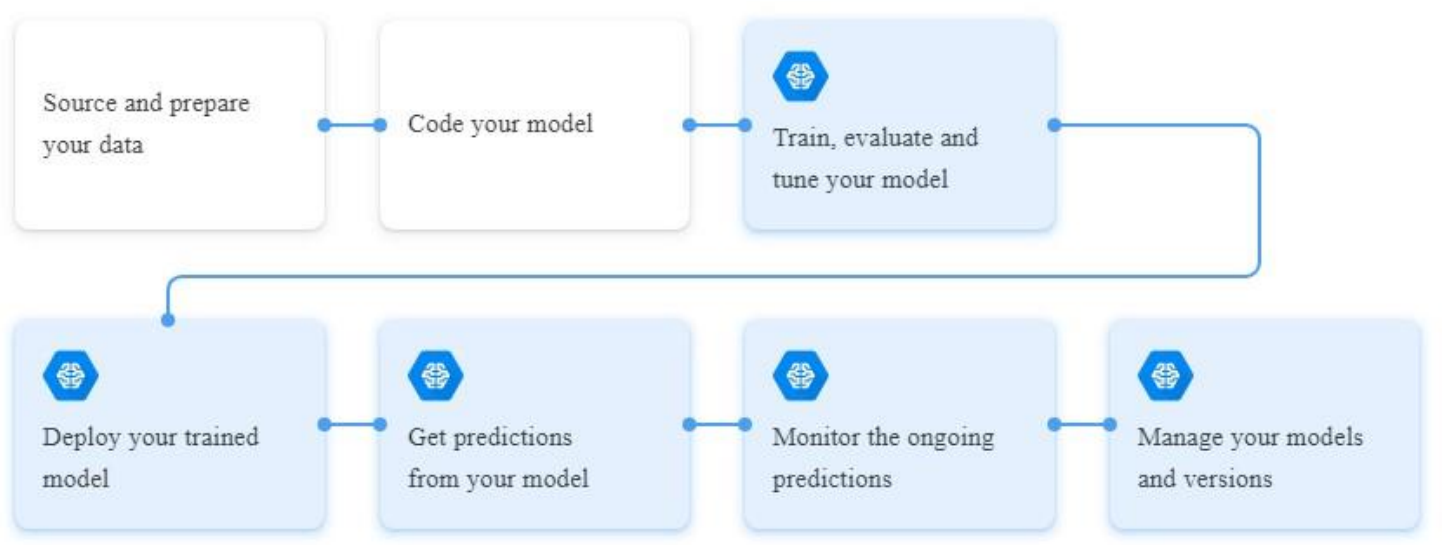

Figure 4 Machine Learning Workflow [8]

Machine learning development process is documented by [8], figure 5 .

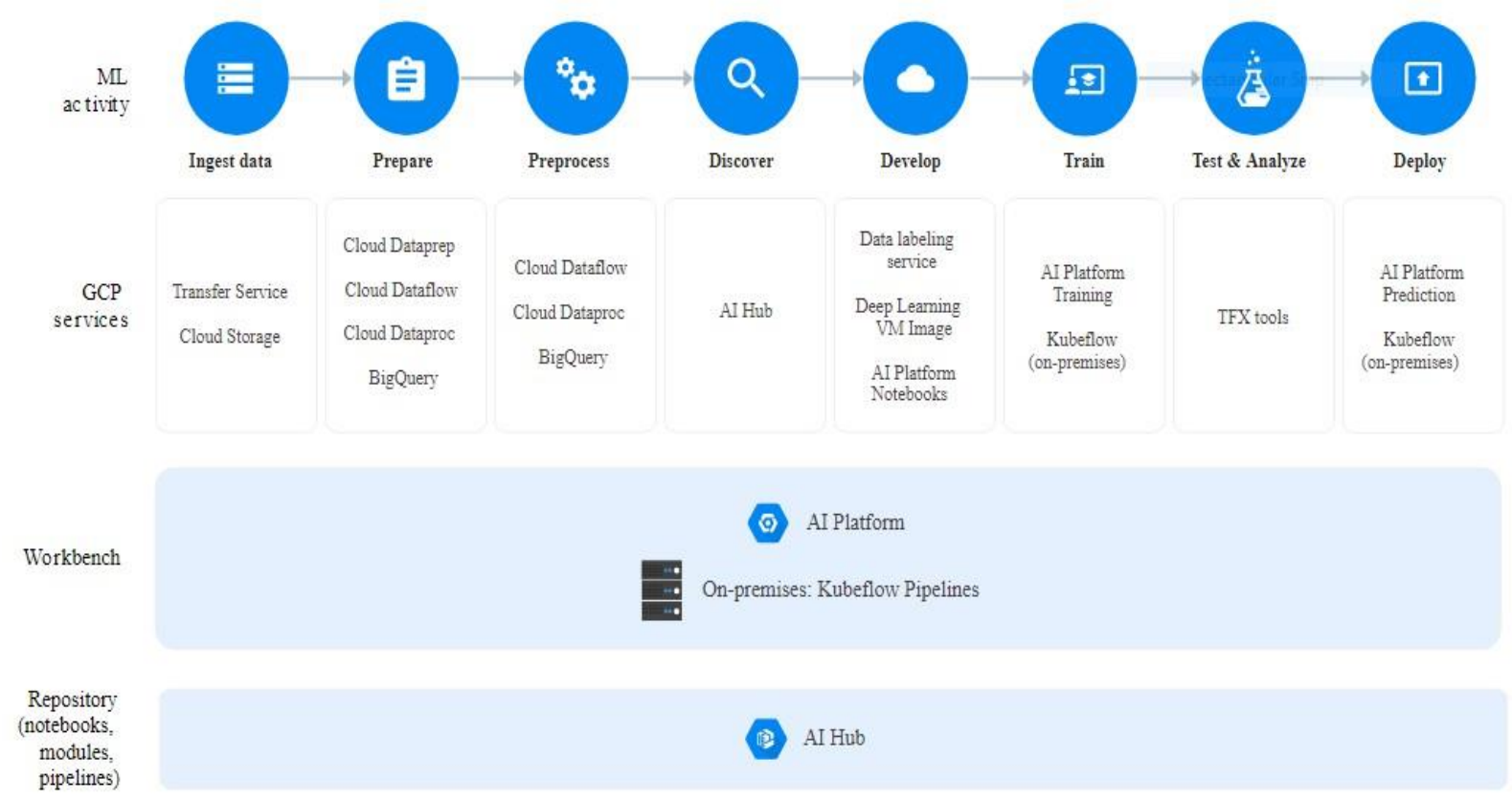

Figure 5. Machine learning development: the end-to-end cycle [8]

In Google Cloud, the AI models are created and deployed by using the Cloud Console, fig. 6 . 


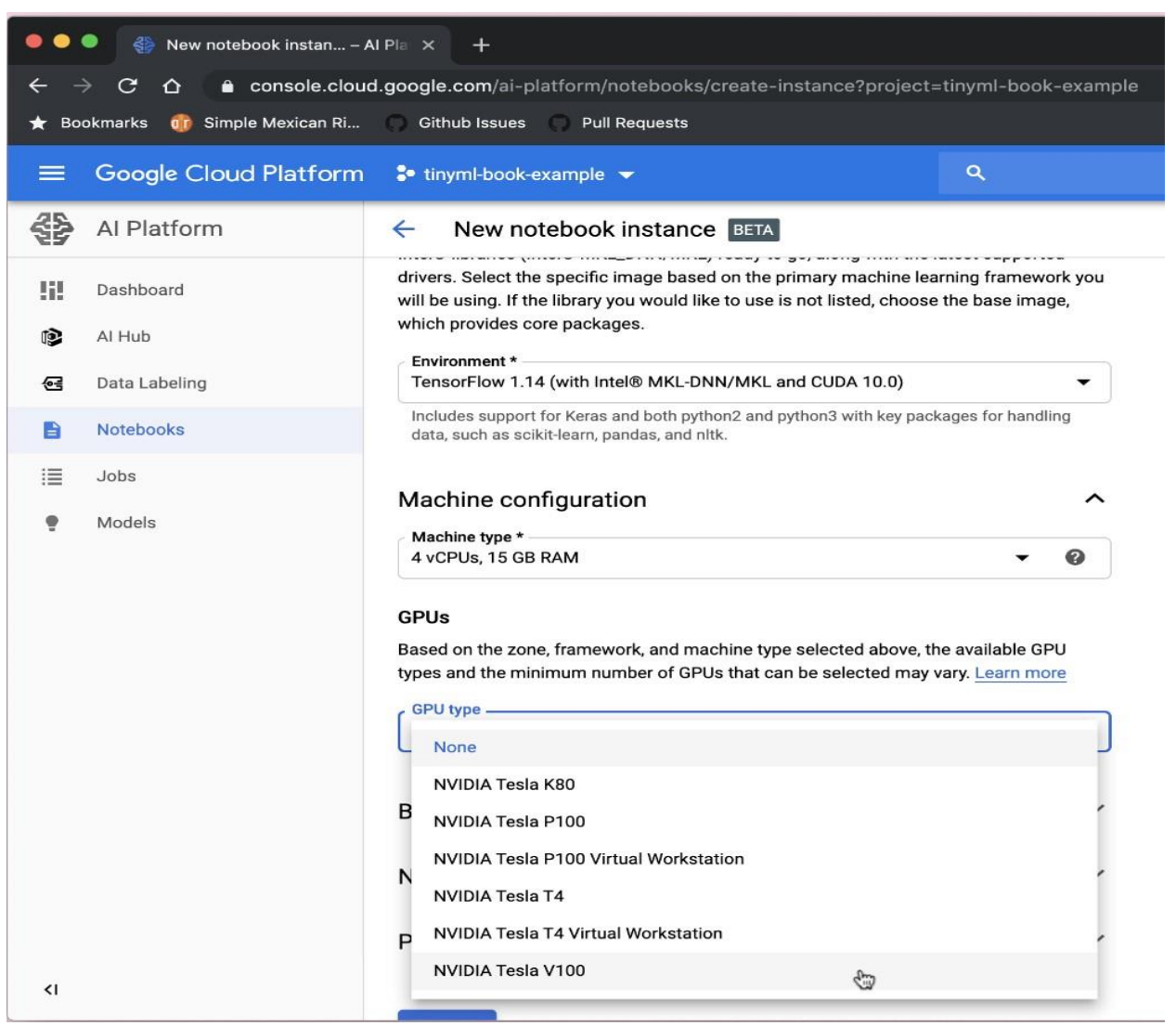

Figure 6. Google Cloud Console

Once designed, an AI model is built and trained. In Google Cloud, an easy way to train a model is to use the Colaboratory (Colab) as a hosted Jupyter notebook service. That notebook allows to write and execute Python code with no setup to be done before.

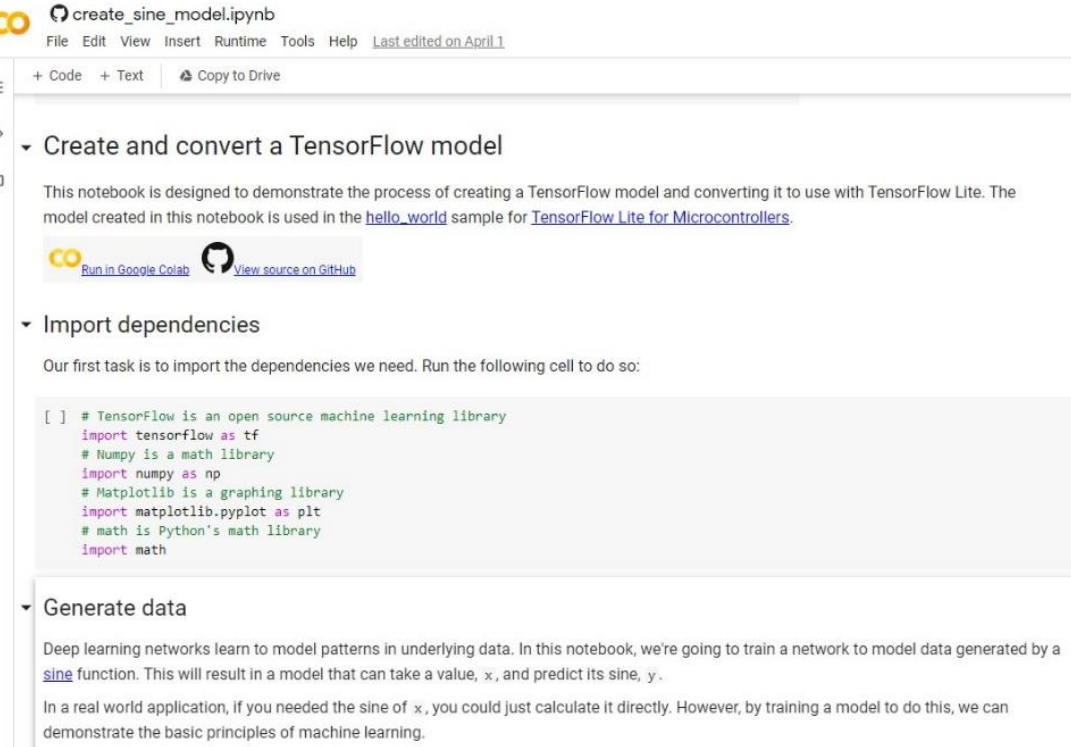

Figure 7. Google Cloud Console [9]

Within the Colab notebook, some tuning operations like enabling GPU training, configuration for training, installing dependencies or loading TensorBoard are performed. TensorBoard is a tool to visualize and track different parameters during the model training, figure 8 . 


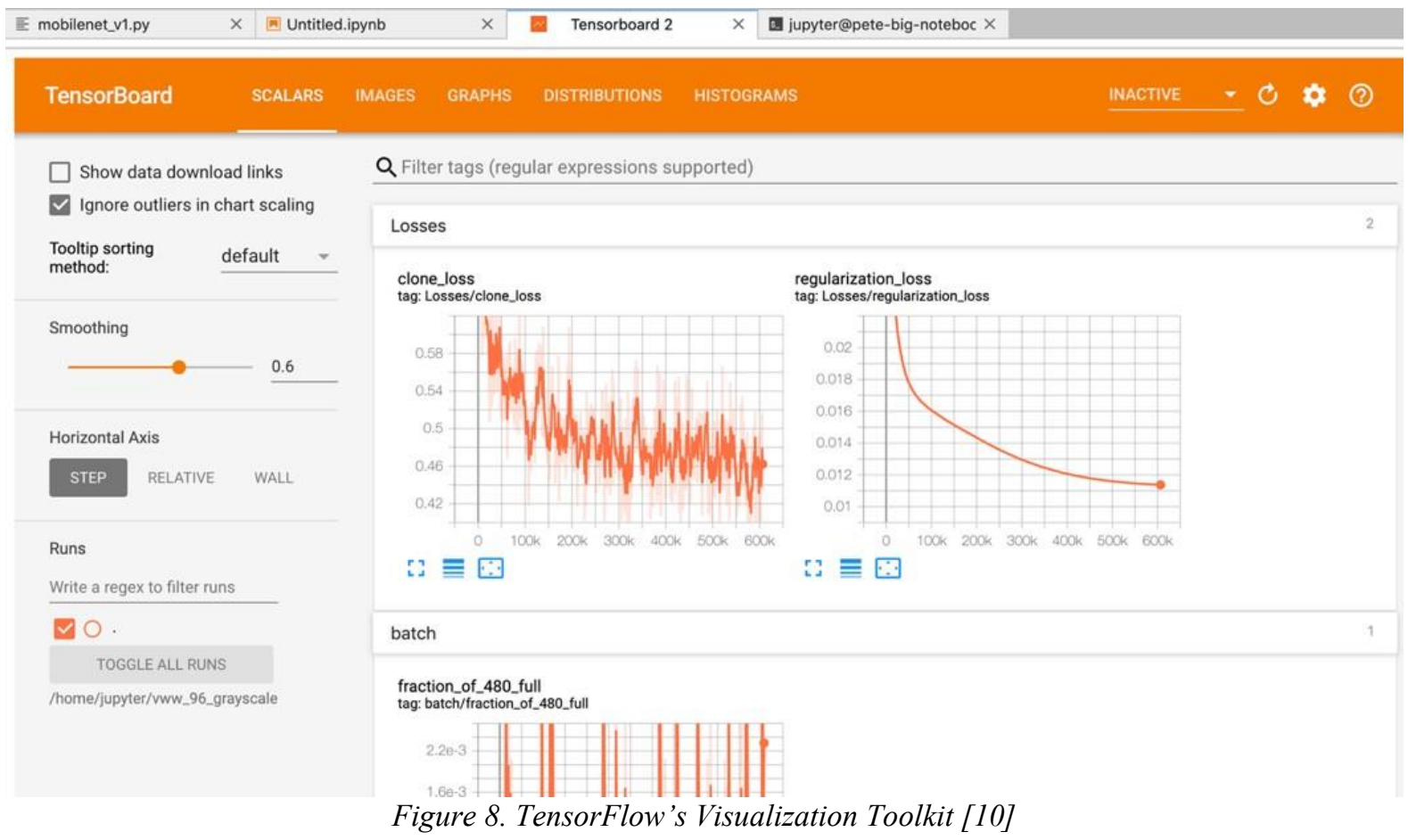

The aspects presented above are common for both classic and tiny ML models. The specificity of a tiny model is that after training some additional operations are made in order to deploy the inference on a certain hardware board [1]:

- Generate the lite model - it converts the frozen model (the output of the training phase) into a model fully quantized to be used with embedded devices. TensorFlow provides the needed Python statements to do that. The resulted model is fitted to the constraints of the target embedded devices.

- Convert the model - it converts the TensorFlow Lite model into a $\mathrm{C}$ source file that it is ready to be loaded for microcontroller.

Deployment of the model on a specific microcontroller is done by using TensorFlow Lite for Microcontrollers. It supports several development boards and can be included in any $\mathrm{C}$ or $\mathrm{C}++$ $11 / 14$ project. After conversion of the model into a $\mathrm{C}$ array, the inference is stored in a readonly memory area. The inference is integrated into a microcontroller code which gets data, performs the ML inference and uses the results.

\section{Machine Learning and Neural Network Inference on the Edge Embedded Devices}

Once the models/neural networks values are trained and stored in storage of the embedded device, then the device will be used for performing various inferences according with the learning output. For instance, if the $\mathrm{CNN}$ - Convolutional Neural Network has been trained for recognizing the voice commands to a home personal assistance such as Amazon Alexa/Google Home Assistant or MobileNet neural network has been trained for image processing in order to detect persons presence, then figure 9 and respectively 10, are presenting the main business logic of using applied TinyML technology. 


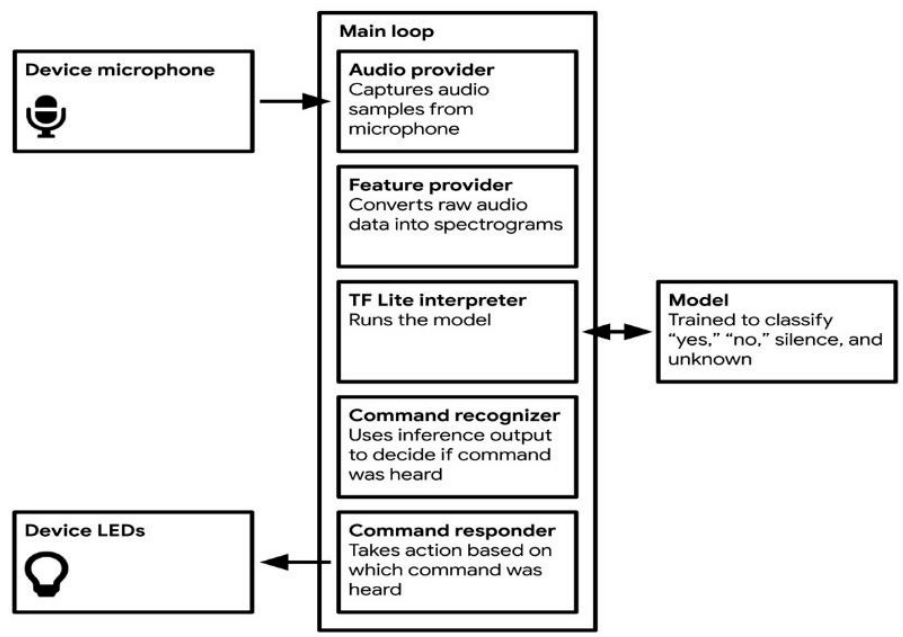

Figure 9. The components of the wake-word application - copyright [1]

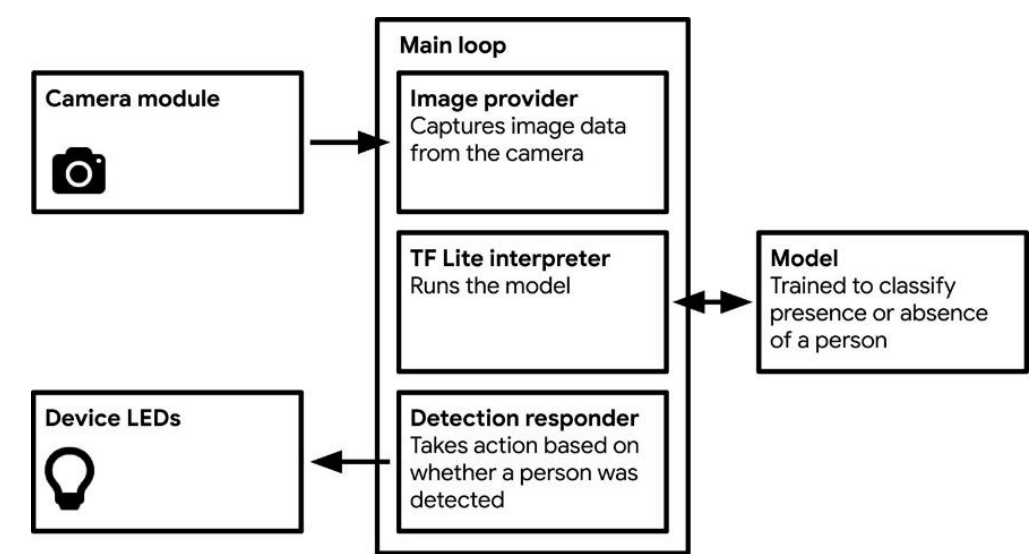

Figure 10. The components of our person detection application - copyright [1]

For both applications, a step-by-step process takes place, as it follows: 1 . The developers obtain an input; and then 2. They Preprocess the input in order to extract proper features to feed into a model; 3. Train the models and prepare them for inferences; (all three steps are performed into an A.I. Cloud or on premise A.I solution); 4. Deploy the model ready to do inference into the embedded boards; 5 . Run inference on the real input which must be preprocessed in real time on the board in order to fit the input layer of the model from the embedded boards (steps 4 and 5 are done inside the IoT Node embedded board).

\section{Conclusions and Cybersecurity Challenges}

The security challenges for deploying neural networks into embedded systems are very important, because of the privacy and integrity of the deployed solutions. Additionally, there are solution in the field with embedded devices which are trained to apply neural networks inferences in sensitive areas such as: monitoring and surveillance cameras, drones visual computing for securing field areas, IoT systems edge security, etc. The exemplified boards such as mbed LPC1768 (mbed OS) or ST Microelectronics STM32F746G embedded devices have 16/32 KB RAM and 128/512 KB of EEPROM and with a process of certification according with an international scheme such as CC-Common Criteria or FIPS, they can be a bullet proof Java Card black boxes, which are very useful in various fields, where deep learning and visual computing are necessary with high degree of security, such as: visual computing, autonomous vehicles and for the institutions digital and physical access. In the future the 
www.conferenceie.ase.ro

authors are targeting to adapt TensorFlow Lite xxd convertor, in order to generate Java Card compliant code, not only $\mathrm{C} / \mathrm{C}++$ source code.

\section{Acknowledgment}

This paper presents results obtained within the PN-III-P1-1.2-PCCDI-2017-0272 ATLAS project ("Hub inovativ pentru tehnologii avansate de securitate cibernetică / Innovative Hub for Advanced Cyber Security Technologies "), financed by UEFISCDI through the PN III "Dezvoltarea sistemului national de cercetare-dezvoltare", PN-III-P1-1.2-PCCDI-2017-1 program.

\section{References}

[1] P. Warden and D. Situnayake. TinyML, Publisher: O'Reilly Media, Inc., Release Date: December 2019, ISBN: 9781492052043.

[2] A. Géron, "Hands-On Machine Learning with Scikit-Learn, Keras, and TensorFlow, 2nd Edition", Chapter 19: "Training and Deploying TensorFlow Models at Scale", Publisher: O'Reilly Media, Inc., Release Date: September 2019, ISBN: 9781492032649.

[3] A. Koul, S. Ganju and M. Kasam, "Becoming a Maker: Exploring Embedded AI at the Edge," in Practical Deep Learning for Cloud, Mobile, and Edge. Publisher: O'Reilly Media, Inc., Release Date: October 2019, ISBN: 9781492034865.

[4] Arduino Nano 33 BLE Sense Development board: https://store.arduino.cc/arduino-nano33-ble-sense-with-headers

[5] SparkFun Edge Development Board Apollo3 Blue: https://www.sparkfun.com/products/15170

[6] Ericsson Blog about TinyML: https://www.ericsson.com/en/blog/2019/12/tinyml-as-aservice

[7] Google A.I. Cloud: https://cloud.google.com/ai-platform

[8] Online Doc for A.I. Cloud: https://cloud.google.com/ai-platform/docs/technical-overview

[9] Google Colab Platform linked to instances containers from A.I. Cloud for running Python Jupyter

Notebooks: https://colab.research.google.com/github/tensorflow/tensorflow/blob/master/tensorflow/lit e/micro/examples/hello_world/create_sine_model.ipynb\#scrollTo=p-PuBEb6CMeo

[10] Tensorflow Online Board: https://www.tensorflow.org/tensorboard 